\title{
Three-dimensional modeling of transport properties in hardened cement paste using metal
} centrifugation-based pore network

Cheng Liu ${ }^{1,2}$, PhD Student, Email: cheng.liu.16@ucl.ac.uk

${ }^{1}$ School of Materials and Science Engineering, Southeast University, Nanjing, 211189, China

${ }^{2}$ Department of Civil, Environmental and Geomatic Engineering, University College London, London, WC1E 6BT, UK

Rusheng Qian 1, PhD Student, Email: qianrs63277@163.com

${ }^{1}$ School of Materials and Science Engineering, Southeast University, Nanjing, 211189, China Guojian Liu ${ }^{3}$, Assistant Professor, Email: liuguojiande@gmail.com

${ }^{3}$ School of Civil Engineering, Suzhou University of Science and Technology, Suzhou, 215011, China Jianming Gao ${ }^{1}$, Professor, Email: jmgao@seu.edu.cn

${ }^{1}$ School of Materials and Science Engineering, Southeast University, Nanjing, 211189, China Mingzhong Zhang ${ }^{2}$, Associate Professor, Email: Mingzhong.zhang@ucl.ac.uk

${ }^{2}$ Department of Civil, Environmental and Geomatic Engineering, University College London, London, WC1E 6BT, UK

Yunsheng Zhang 1,4*, Professor, Email: zhangyunsheng2011@163.com

${ }^{1}$ School of Materials and Science Engineering, Southeast University, Nanjing, 211189, China ${ }^{4}$ Key Laboratory of Disaster Prevention and Mitigation in Civil Engineering of Gansu Province, Lanzhou University of Technology, Lanzhou, 730050, China

\begin{abstract}
:
Accurate prediction of transport properties in cementitious materials remains a challenge since it is difficult to experimentally obtain the realistic three-dimensional (3D) pore network of cement paste. In this study, the 3D pore network of cement paste is quantitatively characterized using Nano X-ray microtomography coupled with the metal centrifugation porosimetry (MCP). First, the MCP technique is carried out to impel the liquid metal into the pore network of cement paste. Subsequently, the 3D pore network of cement paste with the intruded metal is reconstructed using high-resolution X-ray microtomography. Finally, based on the X-ray microtomography images, the transport properties of cement paste including ionic diffusivity and fluid permeability are simulated using a set of lattice Boltzmann transport models. The results show that the metal alloy can significantly enhance the contrast between the pore network and solid phase, and the realistic 3D pore network can be *Corresponding author: Yunsheng Zhang,E-mail: zhangyunsheng2011@,163.com
\end{abstract}


successfully reproduced. For cement paste with water-to-cement $(w / c)$ ratios of 0.5 and 0.6 , the transport properties are dramatically increased with increasing $w / c$ ratio. A similar increasing trend in transport properties can be found for effective porosity of cement paste ranging from $27.56 \%$ to $36.11 \%$.

Keywords: Cement paste; Transport properties; Pore structure; Metal centrifugation porosimetry; Lattice Boltzmann method

\section{Introduction}

Concrete with porous attributes is normally in face of various durability issues that are directly associated with the transport of species, e.g. ions, gas, and water, when exposed to its service environment (Ghasemzadeh and Pour-Ghaz 2015; Jafari Azad et al. 2019; Liu et al. 2020; Yang et al. 2021). The aggressive species can penetrate the interior of concrete via its pore network and reacts with its components, leading to material degradation and reduction in service life for concrete structures. For example, the product expansion of cement hydration, the corrosion of steel-bar, and the carbonization of concrete are highly related to liquid-water, chloride, and $\mathrm{CO}_{2}$ transports, respectively (Garbalinska et al. 2013; Li et al. 2017; Liu et al. 2016). Transport properties including diffusivity and permeability are normally considered as the vital indicators for assessing the potential durability and safety of concrete. Generally, lower transport rates indicate superior concrete durability. As a result, it is vital and necessary to investigate transport properties of concrete.

Concrete at the mesoscale is made up of porous cement paste matrix and non-porous aggregates. Transport properties of concrete are highly dependent on the pore network of cement paste (Song et al. 2019). As such, an accurate characterization of the pore network within cement paste is required for a better understanding and estimating the transport properties of concrete (Qian et al. 2021). Currently, the analysis of the pore network in cement paste mainly focuses on its pore features, e.g. porosity, size, and distribution, which are usually obtained using conventional measurements, e.g. mercury intrusion porosimetry (MIP), Brunaures-Emmitt-Teller adsorption (BET), scanning electron microscope (SEM) (Attari et al. 2016; Kumar and Bhattacharjee 2003; Lapham and Lapham 2019). Among these technologies, MIP is the most widely used approach because of its strengths of the continuous dynamic-range of pore size characterization, easy operation and short testing duration (Li et al. 2017). However, the MIP technology is still questionable due to the ink-bottle effect and certain assumptions (Moro and Böhni 2002). For instance, the pore size is obtained using an assumption of the equivalent circular pore, which enlarges the deviations of experimental data. Similarly, the BET 
technology is coupled with various simplified pore models to investigate pore structure of cement paste (Lapham and Lapham 2019). The BET technology excels at the characterization of small pores but requires a quite long testing period. To improve the accuracy of pore measurement, the SEM technology is used to observe pores in nanometer but only in two-dimension (2D) (Attari et al. 2016). These conventional methods enable us to acquire some pore parameters but cannot obtain the actual $3 \mathrm{D}$ pore network in cement paste.

To tackle the above-mentioned drawbacks, some 3D tomography techniques including X-ray computed tomography (X-CT), focused ion beam/scanning electron microscopy (FIB/SEM), and laser scanning confocal microscopy (LSCM) have been developed to characterise the pore structure of cement paste in 3D. X-CT is employed to obtain the 3D pore structure based on the relative attenuation coefficients of pore and solid phases within the materials (Zeng et al. 2019). However, the current X-CT technology is usually used for the analysis of pores with large size (e.g. foam concrete) due to the low attenuation difference between two phases (She et al. 2018). The insufficient attenuation difference causes trouble in distinguishing the interface between the pore and solids. The discriminations for the two phases are usually accompanied by subjectivity. To this end, the FIB/SEM is applied for the characterisation of 3D pore network in cement paste. During the FIB/SEM operation, FIB first provides a slice cutting with a constant thickness (a few nanometers thickness) on the specimen surface, and then the freshly cut surface is sequentially recorded by SEM (Song et al. 2019). Accordingly, the FIB/SEM image tomograms with equally spaced 2D images are produced, based on which the 3D pore network can be re-constructed (De Winter et al. 2009). However, the FIB/SEM technology is a destructive examination due to the automatic cutting and is quite time-consuming and prohibitive. Lately, an emerging 3D imaging technology which couples LSCM to serial sectioning was proposed to characterise the $3 \mathrm{D}$ pore network within porous materials at a submicron spatial resolution (Yio et al. 2015). This method is associated with the stitching of sequential confocal tomograms according to the phase correlation, leading to the large image volumes without resolution loss. Successful analyses of pore structure using the LSCM technology require that the pore network can be accurately segmented from the images. However, the segmentation of the pore network is still extremely challenging due to the complex boundaries between pore and solid phases within cement paste. Besides, the segmentation procedure is further complicated due to the heterogeneous brightness possibly appearing along with the reconstruction depth of images. The limited contrast between pores and solids within cementitious materials hinders the applications of X-CT, FIB/SEM, and LSCM 
techniques in the accurate characterisation of 3D pore structure of cementitious materials.

To improve the contrast for in-situ characterisation of 3D pore network, various contrast agents, such as Wood's metal (Kaufmann 2010; Willis et al. 1998), mercury (Klobes et al. 1997) and polymethylmethacrylate (Hellmuth et al. 1999), have been recently employed in the characterisation of pore network in porous materials. Wood's metal combined with FIB is usually utilized in clay rock for obtaining its 3D structure (Desbois et al. 2016). Mercury and polymethylmethacrylate combined with X-CT are employed to explore the 3D pore network in crystalline rock (Hellmuth et al. 1999; Klobes et al. 1997). However, the X-CT resolution usually used is not enough for micro pore-scale study. Besides, the mercury has toxic effect on health and ease volatileness at room temperature and thus its use in laboratory is a potential hazard. Although these contrast agents are widely used in the rock fields, they have not been yet broadly used for characterising the $3 \mathrm{D}$ pore structure of cementitious materials. It inspires us to develop the potential advantages of this technique to investigate the $3 \mathrm{D}$ pore network in cement paste and gain new insights into pore features.

Transport properties (diffusivity and permeability) of cement paste are significantly associated with its microstructure, which can be obtained using experimental measurements and numerical simulations (Oslakovic et al. 2010; Patel et al. 2016). Generally, the experimental measurements can be accomplished using natural or electrically accelerated methods (Patel et al. 2016). However, there is a significant difference in the measured values of transport properties from different methods and there is still no agreement on the standardized measurements (Tang and Sorensen 2001). Moreover, the experimental measurements are usually time-consuming and expensive (Liu et al. 2020). It is still a great challenge to accurately measure the transport properties of cement paste. In contrast to experiments, numerical simulation shows more potential for conveniently obtaining the effective transport properties of cement paste, where the influences of different factors can be considered. Different numerical methods including finite difference method (Garboczi and Bentz 1992; Ukrainczyk and Koenders 2014), finite element method (Garboczi and Bentz 1992), lattice Boltzmann method (Zhang et al. 2012) and random walk method (Liu et al. 2019) have been used to simulate the diffusivity and water permeability (Zhang et al. 2013; Banala and Kumar 2017) of virtual hydrating Portland cement pastes generated by CEMHYD3D, HYMOSTRUC3D and $\mu$ ic models, respectively. The simulation results of diffusivity and permeability both show good agreement with experimental data. However, these simulations were carried out only based on virtual 3D pore-structure of cement paste. The predictions using the actual 3D pore structure should be performed to provide a better 
understanding of the transport properties in cement paste.

This study aims to characterise the 3D pore network in cement paste, based on which the transport properties in cement paste including ionic diffusivity and fluid permeability were simulated. To this end, the metal alloy with a low-melting-point is selected as the proper contrast agent for improving the pore resolution by a specific centrifuge device. The metal centrifugation porosimetry (MCP) technology is carried out to impel the metal to intrude the pore network within cement paste. The 3D pore structure of cement paste with the contrast agent is reconstructed using the high-resolution Xray microtomography. The pore structure characteristics including porosity, connectivity, and pore size distribution were quantitatively investigated. Subsequently, the ionic diffusion and fluid permeation through the obtained 3D pore network in cement paste with various water-to-cement (w/c) ratio were simulated using a set of lattice Boltzmann (LB) models for transport and then the ionic diffusivity and fluid permeability were calculated. The simulation results were compared with experimental data.

\section{Materials and Methods}

\subsection{Raw materials}

In this study, Portland cement (Chinese standard Graded P.II 52.5 type) is used, the density and specific surface area of which are $3150 \mathrm{~kg} / \mathrm{m}^{3}$ and $369.60 \mathrm{~m}^{2} / \mathrm{kg}$, respectively. Table 1 presents the chemical composition of cement. The initial and final setting time of cement paste is 132 min and $187 \mathrm{~min}$, respectively. The compressive strength and flexural strength of cement paste under standard curing conditions for $28 \mathrm{~d}$ are $59.60 \mathrm{MPa}$ and 9.20 MPa, respectively.

Table 1 Chemical composition of cement (wt.\%).

\begin{tabular}{llllllllll}
\hline Oxide & $\mathrm{SiO}_{2}$ & $\mathrm{Al}_{2} \mathrm{O}_{3}$ & $\mathrm{Fe}_{2} \mathrm{O}_{3}$ & $\mathrm{CaO}$ & $\mathrm{MgO}$ & $\mathrm{SO}_{3}$ & $\mathrm{Na}_{2} \mathrm{O}$ & $\mathrm{K}_{2} \mathrm{O}$ & LOI \\
\hline Content & 21.35 & 4.67 & 3.31 & 62.60 & 3.08 & 2.25 & 0.11 & 0.65 & 0.95 \\
\hline
\end{tabular}

\subsection{Description-of-the-art: MCP method}

The characterisation of the $3 \mathrm{D}$ pore network consists of three main parts including metal centrifugation intrusion (Fig. 1(a)), X-ray microscopic observation (Fig. 1(b)), and 3D pore network reconstruction (Fig. 1(c)), which were performed with the self-manufactured centrifuge device, the CT scanner and the ImageJ software, respectively.

\subsubsection{Property of the Metal}

In the MCP operation, the metal is employed as the contrast agent, which is composed of a series of elements, i.e. bismuth (Bi, 43.7\% wt.), lead ( $\mathrm{Pb}, 20.6 \%$ wt.), tin ( $\mathrm{Sn}, 8.6 \% w t$.), chromium $(\mathrm{Cr}, 5.8 \%$ $w t$.), indium (In, 20.1\% wt.) and other microelements. The atomic numbers of the elements are higher 
than those contained in cement paste with main elements of silicon $(\mathrm{Si})$, calcium $(\mathrm{Ca})$, oxygen $(\mathrm{O})$, aluminium (Al). Correspondingly, the specific gravity of the metal is $9.4 \mathrm{~g} / \mathrm{cm}^{3}$, which is also higher than that of cement paste. Generally, the contrast agent with a higher density is conducive to acquire a greater centrifuge force during the MCP operation, which is the key to successfully intrude the metal into the pore space of cement paste. Besides, the metal has a low melting point of only $47^{\circ} \mathrm{C}$, which can guarantee the liquid state of the metal during the intrusion progress. To decrease the energy cost and avoid the decomposition of ettringite, temperature $65^{\circ} \mathrm{C}$ was chosen in the experiment (slightly higher than the melting point of the metal alloy used). In this study, the temperature was also set as $65{ }^{\circ} \mathrm{C}$ for obtaining the contact angle of the metal in contact with cement paste, which was executed by High-Temperature Vacuum Contact Angle Tester (Qian et al. 2018). During the testing, the metal and cement paste were first heated to reach temperature $65^{\circ} \mathrm{C}$ in the heating system of the tester and then their contact angle was recorded by the tester. The contact angle was measured to be $110^{\circ} \sim 130^{\circ}$, which is close to the contact angle of mercury to paste $\left(117^{\circ} \sim 140^{\circ}\right)$ (Kumar and Bhattacharjee 2003).

\subsubsection{Intrusion Procedure of Metal Centrifugation}

Cement paste specimens $(w / c=0.5,0.6$ and 1.0$)$ were prepared to form cavity shape. The cavity formation aims to hold the molten metal alloy using a specific device (Fig. 1(a)). First, a test tube was utilized to store the fresh cement paste. Then, a specific plug, i.e. the bar-pipe plug, (Fig. 1(a), was subsequently pressed down to produce the cavity. After the cement paste was hardened, the specific plug was removed out carefully and the cement paste was then cured at a standard curing room $(20 \pm$ $\left.1{ }^{\circ} \mathrm{C}, 95 \% \mathrm{RH}\right)$ for $28 \mathrm{~d}$. Finally, the hardened cement paste specimens were terminated hydration using ethyl alcohol to soaking the specimens for $3 \mathrm{~d}$ and then dried in an air oven at $65{ }^{\circ} \mathrm{C}$ temperature until the constant mass.

After the cavity specimen was dried, the specimen was transmitted into the self-made thermalinsulation centrifuge tube, where the metal alloy can be impelled into the pore space of the specimen using a centrifuge machine. Detailed operating steps were described as follows. First, the metal and the cavity specimen were severally heated $\left(65^{\circ} \mathrm{C}\right)$ until the metal became molten. Then, the molten metal was poured into the cavity of the specimen and the specimen was tightly coated with hermetic polystyrene for heat preservation. After that, the specimen was carefully placed in the centrifuge machine for the subsequent centrifuge operation at $4000 \mathrm{r} / \mathrm{min}$ for $0.5 \mathrm{~h}$. Finally, the specimen was cooled at room temperature. Due to the density of the metal alloy $\left(9.40 \mathrm{~g} / \mathrm{cm}^{3}\right)$ higher than that of the hardened cement paste, the former can acquire higher centrifuge force than the latter under the same 
conditions. Thus, the cavity shape of cement paste for holding the molten metal is the guarantee for the successful intrusion of the molten metal into pore network.

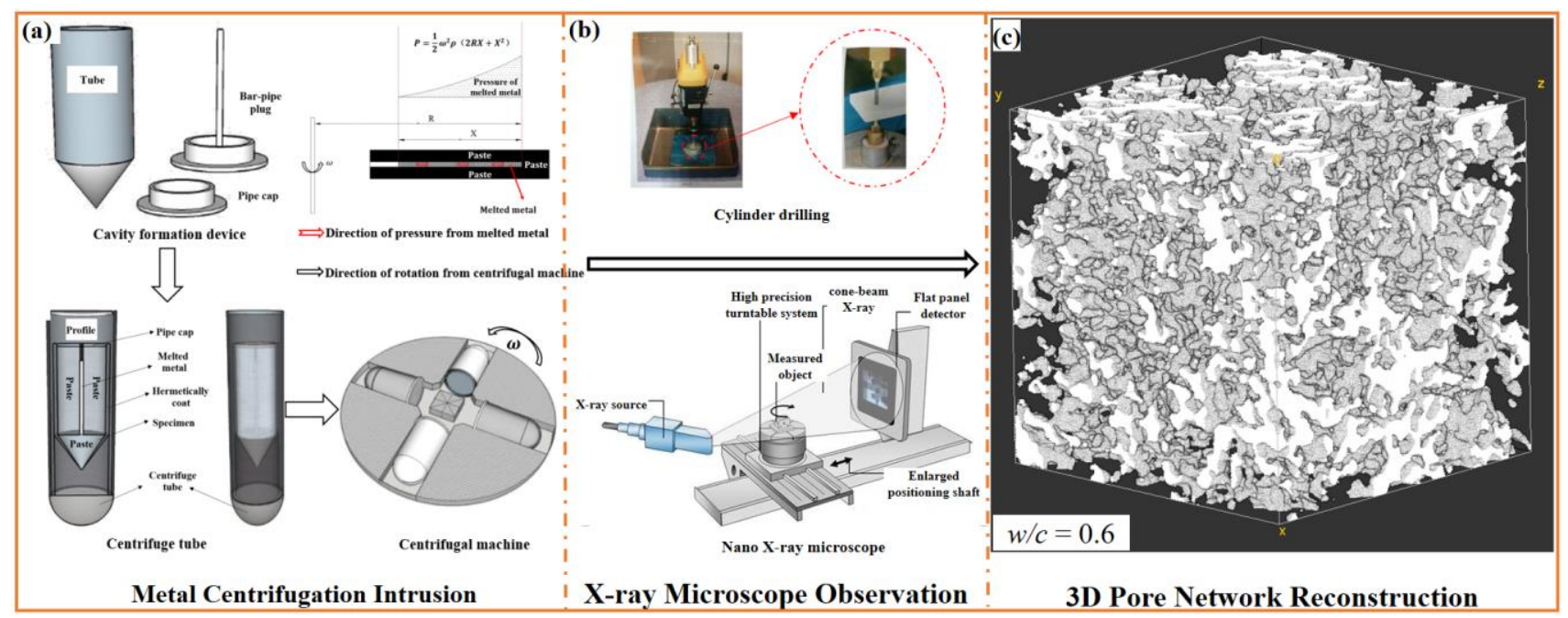

Fig. 1. Flow chart of the MCP method: (a) specimen preparation and metal intrusion; (b) CT images and (c) re-constructed 3D pore network of cement paste with $w / c$ ratio of 0.6 .

\subsubsection{Scanning electron microscope and CT scan}

SEM combined with back scattered electron (BSE) was employed as an auxiliary technique to prove that the pore space of the tested specimen was successfully intruded by the metal alloy. SEM-BSE technology is usually used for obtaining the $2 \mathrm{D}$ pore structure. In this study, the $3 \mathrm{D}$ characterisation of pore network was provided by the CT scan technique. The specimen size significantly determines the voxel size of the images, which further affects the pore-size observed. Thus, the smaller size of the sample can facilitate the acquisition of the finer pore within the sample and vice versa. As a result, the cylindrical sample with a diameter of around $1 \mathrm{~mm}$ was drilled out of the cavity specimen for CT testing in this study. All specimens were scanned using the CT scanner for $5 \mathrm{~h}$ under the same station. The images with a pixel size of $1.0 \mu \mathrm{m}$ were exported for reconstructing the pore network in cement paste.

\subsubsection{D pore network reconstruction}

After a series of alignment, cropping and filtering, the segmentation of image tomograms can be achieved. This process can be accomplished using a software ImageJ with its built-in parameters. First, the binarization of image tomograms should be pre-executed. After that, the grey value threshold should be selected for distinguishing the metal alloy (pores) and the solid phases. The threshold can be determined using an inflexion-point method at the cumulative curve of the grey value histogram (Wong et al. 2006; Zhang et al. 2013). Then, the parameters of pore structure 
including porosity, size, and distribution can be obtained for the specimens with w/c ratios of $0.5,0.6$ and 1.0 .

\subsubsection{Representative elementary volume}

To simulate the transport properties, the cement paste executed in this study should be sufficiently representative and thus the representative elementary volume (REV) needs to be determined first, where the property fluctuations of specimens considered can be neglected (Rozenbaum and du Roscoat 2014; Yio et al. 2017). As illustrated in Fig. 2, the change of REV size considered induces the property fluctuations of pore network within specimen during observation, e.g. porosity (Pichler et al. 2013; Yio et al. 2017). This is the so-called "microscopic effect", where the property fluctuations of interest should be considered. The fluctuations of pore characteristics can be precisely quantified for identifying the pores affecting the transport properties in cement paste (Song et al. 2020). Similar to previous studies, the REVs of cement paste were deliberately optimised by increasing the specimen size (Kanit et al. 2003; Song et al. 2020; Yu et al. 2018). Yio et al. (2017) showed that most pore characteristics of cement paste are independent of the specimen size beyond $60^{3} \mu \mathrm{m}^{3}$ (i.e. $\mathrm{REV}=60^{3}$ $\mu \mathrm{m}^{3}$ ). Besides, the REV was found to be $100^{3} \mu \mathrm{m}^{3}$ from the statistical analysis of porosity with a CoV of $5 \%$ (Yio et al. 2017). In this study, a REV of $200^{3} \mu \mathrm{m}^{3}$ corresponding to $200 \times 200 \times 200$ voxels was obtained to have the representative meaning of cement paste in terms of macroscopic properties.

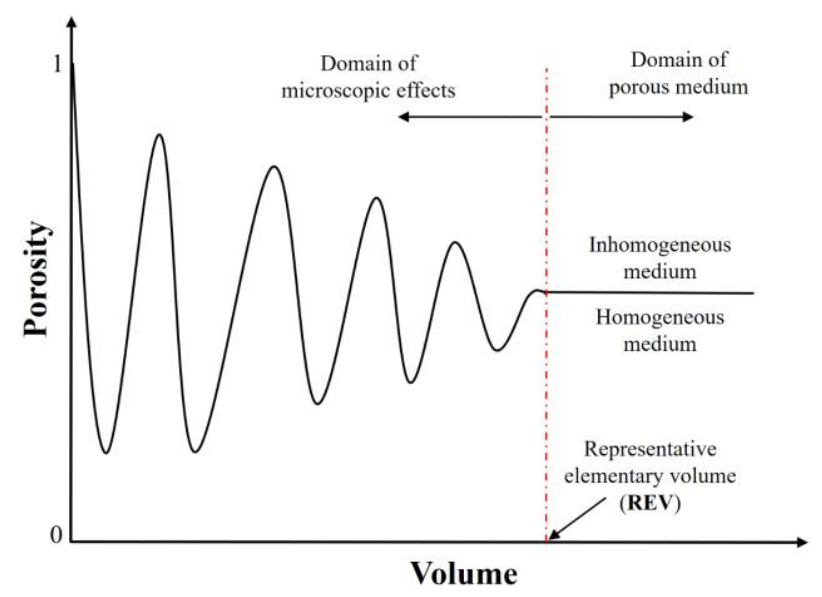

Fig. 2. Determination of REV based on the change in porosity of cement paste against the specimen volume.

\section{Lattice Boltzmann modelling of transport properties}

Here, the LB models for ionic diffusion and fluid permeation are developed. LB method is derived from the kinetic Boltzmann equation that handles the fluid as a polymer of artificial particles and simulates the microscopic characteristics of fluid based on the inter-particle collisions. According to the continuous Boltzmann equation, the fluid particles can move in all directions at the microscopic level. LB method is used to approximately describe the continuous Boltzmann equation through two 
steps: the physical space of the specimen is discretized into a set of equally spaced lattice nodes, and the velocity space of fluid is discretized into a finite set of microscopic velocity vectors (Zhang et al. 2013). LB method has been successfully employed to simulate fluid flow and ionic diffusion in porous media and already achieved considerable success because of its easy implementations of boundary conditions, complex geometry as well as multiple inter-particle interactions (Pan et al. 2006). The LB modelling procedures are described in detail below.

\subsection{Lattice Boltzmann method}

For single-relaxation-time (SRT) collision operator, the particle distribution evolution satisfies with the following discrete lattice Boltzmann equation:

$$
f_{i}\left(\mathbf{x}+\mathbf{e}_{i} \delta t, t+\delta t\right)-f_{i}(\mathbf{x}, t)=-\frac{1}{\tau}\left[f_{i}(\mathbf{x}, t)-f_{i}^{e q}(\mathbf{x}, t)\right]
$$

where $f_{i}$ and $f_{i}^{e q}$ represent the functions of particle distribution under non-equilibrium and equilibrium conditions respectively at location $\mathbf{x}$ and time $t$, subscript $i=0,1, \ldots(b-1)$ denotes the velocity direction where $b$ is the number of the discrete lattice velocity direction, $\mathbf{e}_{i}$ is the discrete lattice velocity, $\delta t$ is the lattice time step, and $\tau$ is the non-dimensional relaxation time.

To acquire the high calculation accuracy, a cubic lattice model with 19 discrete velocity directions (D3Q19) as indicated in Fig. 3(a) is normally used to simulate the transport phenomenon in 3D porous media. The corresponding lattice velocity vector of the D3Q19 model can be expressed as:

$$
\mathbf{e}_{i}=\left[\begin{array}{ccccccccccccccccccc}
0 & 1 & -1 & 0 & 0 & 0 & 0 & 1 & 1 & 1 & 1 & 0 & 0 & -1 & -1 & -1 & -1 & 0 & 0 \\
0 & 0 & 0 & 1 & -1 & 0 & 0 & 1 & -1 & 0 & 0 & 1 & 1 & 1 & -1 & 0 & 0 & -1 & -1 \\
0 & 0 & 0 & 0 & 0 & 1 & -1 & 0 & 0 & 1 & -1 & 1 & -1 & 0 & 0 & 1 & -1 & 1 & -1
\end{array}\right]
$$

For pure diffusion, $f_{i}^{e q}$ can be calculated as:

$$
f_{i}^{e q}(\mathbf{x}, t)=\frac{1}{b} C(\mathbf{x}, t)
$$

where $C(\mathbf{x}, t)$ represents the mass concentration and is equal to $\sum_{i=1}^{N} f_{i}(\mathbf{x}, t)$.

As the discrete velocity directions decrease from D3Q19 to D3Q7, it can still provide an accurate simulation result for the pure diffusion of cement paste (Jeong et al. 2008). To improve the efficiency of computation and decrease the computational cost, the D3Q7 model instead of D3Q19 is employed as the cubic lattice model in this study, as depicted in Fig. 3(b). The discrete lattice velocity for the D3Q7 model can be expressed as:

$$
\mathbf{e}_{i}=\left[\begin{array}{ccccccc}
0 & 1 & -1 & 0 & 0 & 0 & 0 \\
0 & 0 & 0 & 1 & -1 & 0 & 0 \\
0 & 0 & 0 & 0 & 0 & 1 & -1
\end{array}\right]
$$


For the D3Q7 model, the $\tau$ value for pure diffusion in lattice units $(\widetilde{D})$ depends on the diffusivity:

$$
\widetilde{D}=\frac{2}{7}\left(\tau-\frac{1}{2}\right) v^{2} \delta t
$$

where $v$ denotes the diffusion speed in the lattice model that is equal to $\delta x / \delta t$ where $\delta x$ is the lattice space. Generally, $v$ can be selected at any value between 0.5 and 2 (Wang et al. 2007).

Regarding permeation, $f_{i}^{e q}$ can be described as:

$$
f_{i}^{e q}(\mathbf{x}, t)=\omega_{i} \rho(x, t)\left[1+\frac{\mathbf{u}^{e q} \mathbf{e}_{i}}{c_{s}^{2}}+\frac{\left(\mathbf{u}^{e q} \mathbf{e}_{i}\right)^{2}}{2 c_{s}^{4}}-\frac{\mathbf{u}^{e q^{2}}}{2 c_{s}^{2}}\right]
$$

where $\rho(x, t)$ and $\mathbf{u}(\mathbf{x}, t)$ represent the macroscopic density and velocity, respectively, $\mathbf{u}^{e q}$ is the equilibrium velocity, $c_{s}$ is the sound lattice speed that equal to $\frac{\delta x}{\sqrt{3} \delta t}, \omega_{i}$ denotes the weight factor which is associated with the $\boldsymbol{i}_{\text {th }}$ direction as described by:

$$
\omega_{i}=\left\{\begin{array}{rr}
\frac{1}{3} & i=0 \\
\frac{1}{18} & i=1,2, \ldots, 6 \\
\frac{1}{36} & i=7,8, \ldots 18
\end{array}\right.
$$

$\rho(x, t)$ and $\mathbf{u}(\mathbf{x}, t)$ can be further expressed as:

$$
\begin{gathered}
\rho(x, t)=\sum_{i=1}^{N} f_{i}(\mathbf{x}, t) \\
\rho(x, t) \mathbf{u}(\mathbf{x}, t)=\sum_{i=1}^{N} f_{i}(\mathbf{x}, t) \mathbf{e}_{i}
\end{gathered}
$$

where $\rho(x, t) \mathbf{u}(\mathbf{x}, t)$ is the momenta of fluid elements.

In this study, the applied boundary conditions for LB modelling of transport properties include the inlet/out boundary condition at inlet and outlet of the computational domain ( $x$-direction in this study), periodic boundary conditions for the other four boundaries ( $y$-and $z$-direction), and half-way bounce-back condition at the interface of transport and non-transport phases inside the domain. The implementation of boundary conditions follows a similar procedure to that reported in the literature (He et al. 1997).

Each step of LB simulation consists of a collision step and a streaming step, which are demonstrated in Eqs. (10) and (11), respectively:

$$
\begin{gathered}
\bar{f}_{i}(\mathbf{x}, t)-f_{i}(\mathbf{x}, t)=-\frac{1}{\tau}\left[f_{i}(\mathbf{x}, t)-f_{i}^{e q}(\mathbf{x}, t)\right] \\
f_{i}\left(\mathbf{x}+\mathbf{e}_{i} \delta t, t+\delta t\right)=\bar{f}_{i}(\mathbf{x}, t)
\end{gathered}
$$

where $f_{i}$ and $\bar{f}_{i}$ represent the pre-collision and post-collision states of the particle distribution, respectively. 

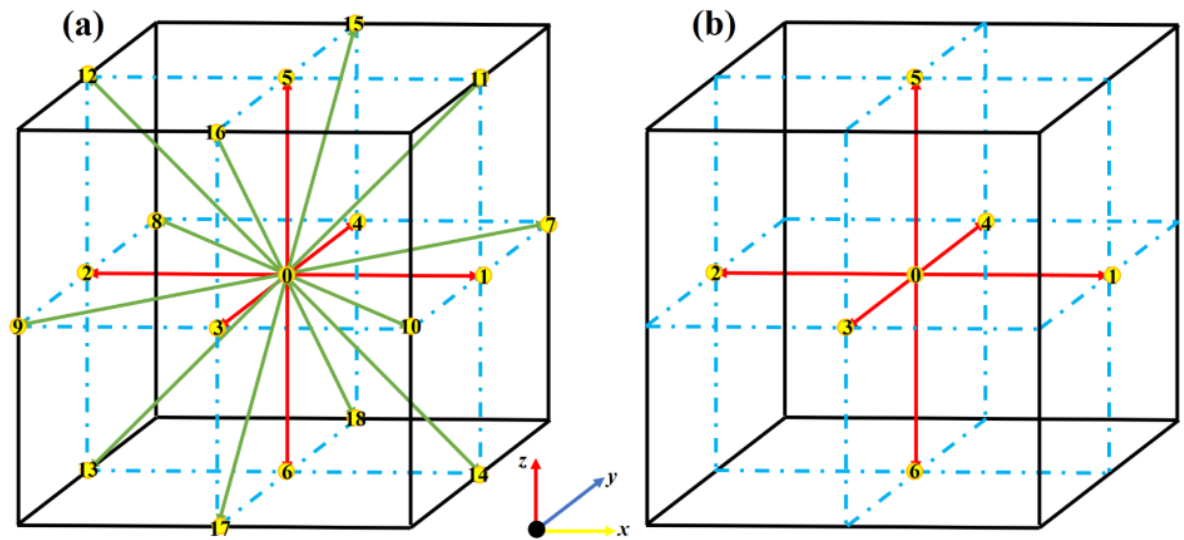

Fig. 3. Cubic lattice model: (a) D3Q19 and (b) D3Q7.

\subsection{Determination of transport properties}

To achieve the steady state, the criterion is defined as the convergence of the average ionic concentration for diffusion and fluid velocity for permeation at three consecutive time steps less than $10^{-8}$ within the whole domain. The physical diffusivity of a porous medium $\left(D_{e}\right)$ can be described as:

$$
\frac{D_{e}}{D_{0}}=\frac{\widetilde{D}_{e}}{\widetilde{D}_{0}}=\frac{\tilde{J}}{\tilde{J}_{0}}
$$

where $D_{0}$ and $\widetilde{D}_{0}$ denote the physical and lattice diffusivities in the pore, respectively, $D_{e}$ and $\widetilde{D}_{e}$ are the physical and lattice diffusivities of the porous medium, respectively, $\tilde{J}$ is the diffusive flux via the porous medium, which can be calculated using Eq. (13), and $\widetilde{J_{0}}$ is diffusive flux across a water-saturated pore space with the same size as the porous medium.

$$
\tilde{J}=\sum_{i} \mathbf{e}_{i} f_{i} \frac{\tau-0.5}{\tau}
$$

The permeability of a porous medium can be obtained according to Darcy's law by:

$$
\tilde{\kappa}=\zeta \frac{N_{x}}{\Delta \rho} \frac{1}{N_{x} N_{y} N_{z} \delta x^{3}} \sum_{i=1}^{N_{x}} \sum_{j=1}^{N_{y}} \sum_{k=1}^{N_{z}}\left\langle u_{x}(i, j, k)\right\rangle
$$

where $\tilde{\kappa}$ is the lattice (or dimensionless) permeability of the porous medium, $\zeta$ is the kinematic viscosity of the fluid as $\zeta=c_{S}^{2}(\tau-0.5), \Delta \rho$ is the density difference of fluid between the inlet and outlet, $N_{x}, N_{y}$, and $N_{z}$ are the total number of nodes along $x$-, $y$ - and $z$-direction, $\left\langle u_{x}\right\rangle$ is the average velocity of the fluid along $x$-direction. The dimensionless permeability can be converted into physical permeability ( $\kappa)$ using Eq. (15).

$$
\kappa=\tilde{\kappa} R_{0}^{2}
$$

where $R_{0}$ is the spatial resolution of the medium. In this study, the spatial resolution in the microstructure of cement paste is $1 \mu \mathrm{m} /$ voxel, i.e. $R_{0}=10^{-6} \mathrm{~m}$.

\section{Results and discussion}

\subsection{Image analysis}




\subsubsection{D pore network}

To ensure that the pore space in cement paste is successfully intruded during the MCP operation, the specimens were scanned using SEM-BSE and subsequently CT. As the successful intrusion of the metal occurs, the pores within cement paste can be highlighted as the metal elements (mainly $\mathrm{Bi}, \mathrm{Pb}$, $\mathrm{Sn}, \mathrm{Cr}, \mathrm{In}$ ) are heavier than cement elements (mainly $\mathrm{Si}, \mathrm{Ca}, \mathrm{O}, \mathrm{Al}$ ). In the SEM image, these significantly various components can result in considerably different grey levels (Qian et al. 2018). The brightness domain (high grey levels) of the image indicates that the pore network is successfully intruded by the molten metal. This can be verified using the BSE mode, where the domains with red "+" identification are analyzed for elements, as shown in Fig. 4(a). According to the analysis of the energy spectrum, significant contents of specific elements such as Bi and $\mathrm{Sn}$ can be detected, suggesting again that the melted successfully intrudes into the pore space. The brightness space represents the pore areas occupied by the metal. As seen in Fig. 4(b), the pore space in cement paste can be identified using X-ray microtomography. Similarly, the contrast of grey values is successfully improved for distinguishing the pore and solid spaces in the images because of the presence of the metal with heavy elements.
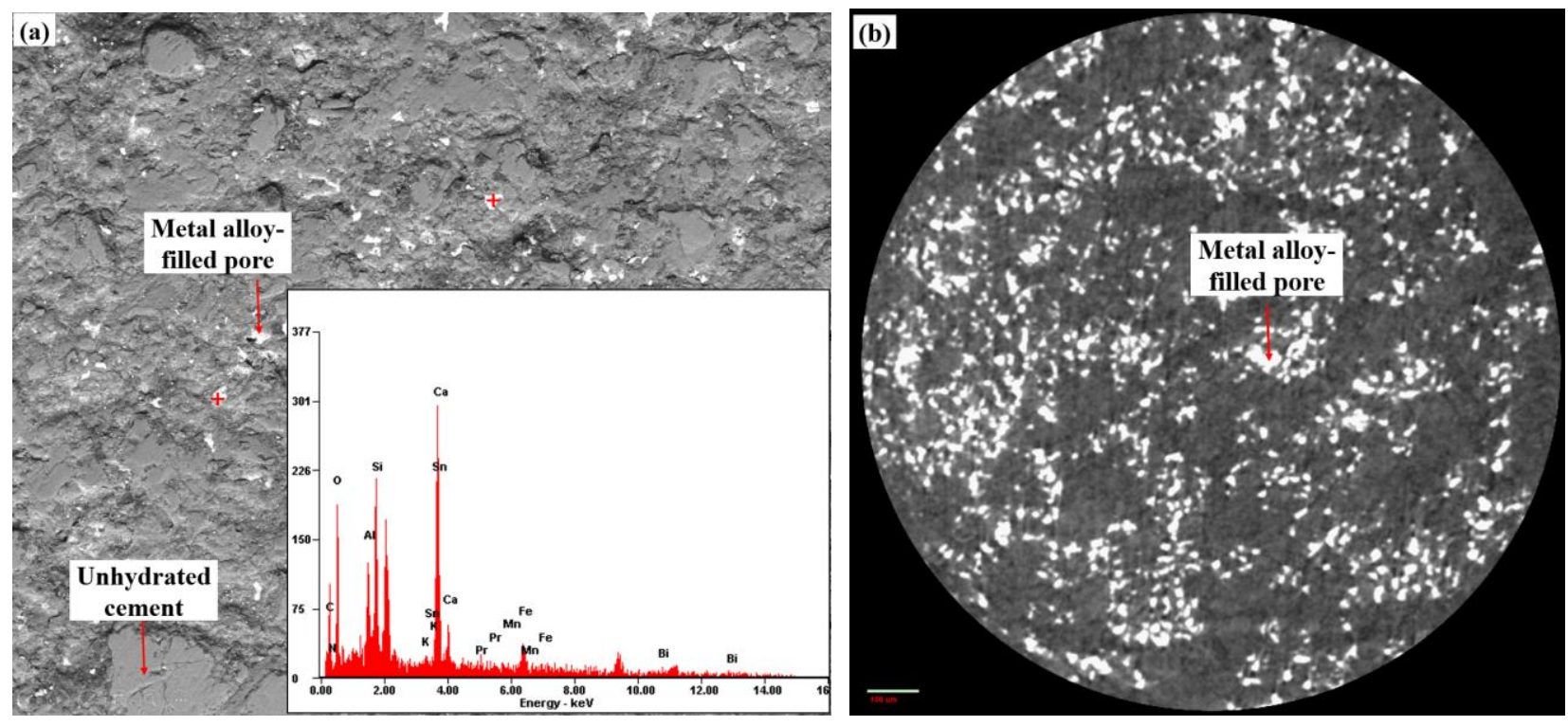

Fig. 4. Images of cement paste $(w / c=0.5)$ obtained using (a) SEM-BSE and (b) X-ray microtomography.

\subsubsection{Reconstitution of 3D microstructure}

Fig. 5 shows the 3D microstructure of cement paste with $w / c=0.5,0.6$ and 1.0. The threshold can be determined using the inflexion-point method at the cumulative curve of the grey value histogram, as illustrated in Figs. 5(a b). Fig. 5(c) depicts the spatial distribution of the pores (the metal alloy) in cement paste. It can be observed that with the increase of $w / c$ ratio from 0.5 to 1.0 , the porosity is 
significantly increased. Meanwhile, the increase of $w / c$ ratio can lead to an increase in the interconnected porosity, which can be mainly attributed to the higher initial porosity and less hydration products in cement paste with a higher $w / c$ ratio.

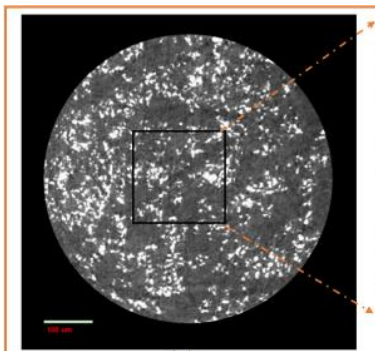

(a)

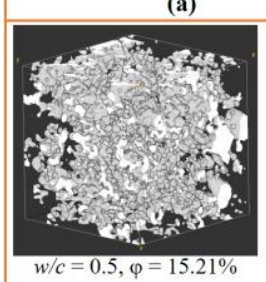

$w / c=0.5, \varphi=15.21 \%$

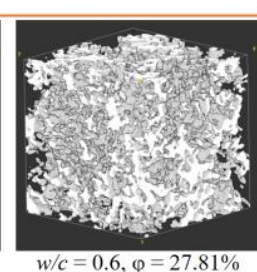

(c)

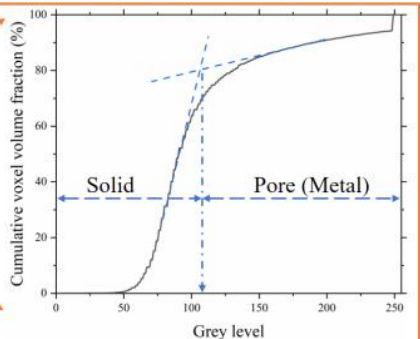

(b)

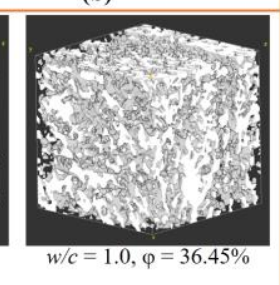

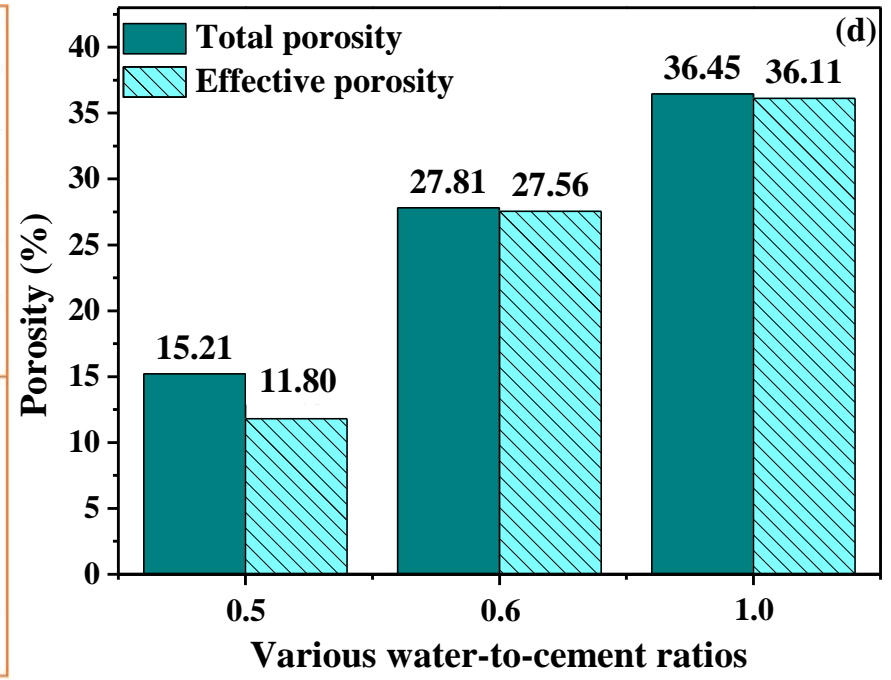

Fig. 5. Grey value thresholding between pores and solid phases based on the grey value histogram (a) and

(b); 3D pore network of cement paste with various $w / c$ ratios (c); total porosity and effective porosity of cement paste with various $w / c$ ratios (d).

\subsection{Pore structure characteristics}

\subsubsection{Porosity}

Porosity of cement paste can be calculated as the pore volume (metal volume) over the total body volume (Hou et al. 2019; Li et al. 2019). Among the main pore structure characteristics, porosity is the direct factor that affects the properties of cementitious materials, such as strength, transport properties and durability. The porosity can be divided into two groups including total porosity and effective porosity, which can be calculated as the ratio of the pore volume and the interconnected pore volume over the specimen volume, respectively. Based on CT images, the 3D pore structure of cement paste composed of pore voxels can be obtained. When the pore voxels face each other and reach into the specimen surface, they are defined as interconnected pores and the corresponding porosity is defined as effective porosity. The interconnection of pores was evaluated using ImageJ. By contrast to the total porosity, the fluid transport in cementitious materials is more significantly associated with effective porosity. As expected, the porosity of cement paste gradually increases from $15.21 \%$ to $36.45 \%$ with increasing $w / c$ ratio from 0.5 to 1.0 (Fig. 5(d)), which can be ascribed to the fact that the high $w / c$ ratio can lead to an increase in the thickness of water coating on the cement particle surfaces, which expands the interspace and thus leaves the coarser capillary pores (Esfandiari and Loghmani 
2019). The greater water content in cementitious materials with a higher $w / c$ ratio is consumed and formed as larger pore space compared to that with a lower $w / c$ ratio. Correspondingly, less hydration products can be generated to fill the pore in cementitious materials with a higher $w / c$ ratio. These result in an increase in the porosity of cementitious materials with a high $w / c$ ratio. Besides, with the increase of total porosity from $15.21 \%$ to $36.45 \%$, the effective porosity also rises from $11.80 \%$ to $36.11 \%$ because of the increase of pore connectivity. The effective porosity is found to be closer to the total porosity for the cement paste with a higher $w / c$ ratio.

\subsubsection{Pore size distribution}

Transport properties of cementitious materials are significantly affected by the pore size distribution of cement paste. Fig. 6 shows the pore size distribution of cement paste determined using MCP. Due to the irregularity of the actual pores, an equivalent pore size was adopted to calculate the pore size distribution from the 3D images (Qian et al. 2018). First, the pore voxels were highlighted and then the voxels in each pore were separately summed up for calculating the equivalent pore size. It can be observed that the main pore size is between 0 and $20 \mu \mathrm{m}$. The increase in $w / c$ ratio leads to an increase in pore diameter of cement paste. For example, for the pores with a size less than $10 \mu \mathrm{m}$, the volume fractions are $95 \%, 80 \%$ and $70 \%$ for cement past with $w / c=0.5,0.6$ and 1.0 , which follows the expectation since the increasing $w / c$ ratio can lead to a larger pore space between cement particles during the initial mixture stage and less hydration products can be formed to fill the pore space in cement paste with a higher $w / c$ ratio.

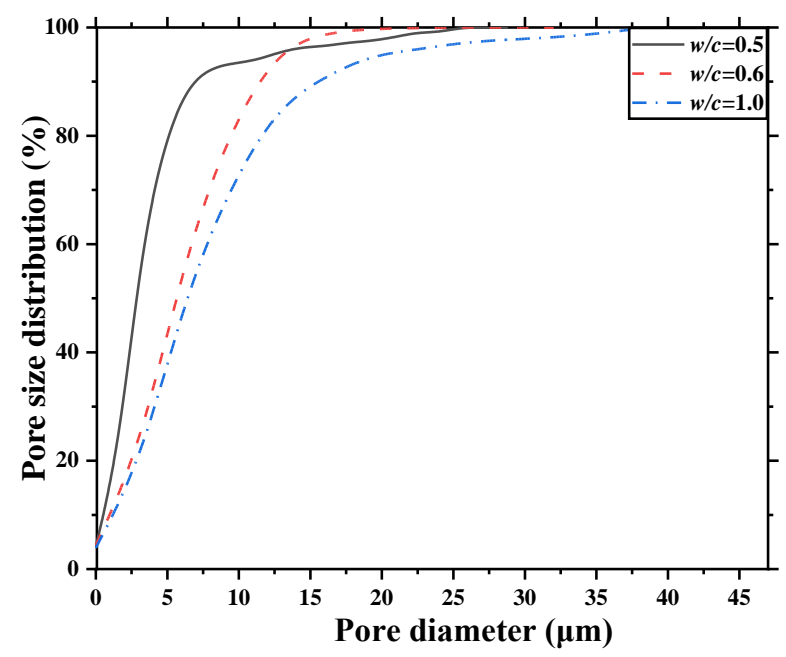

Fig. 6. Pore size distribution of cement paste with various $w / c$ ratios.

\subsection{Simulation results of transport properties}

The LB models for diffusion and permeation were applied to simulate the ionic diffusivity and water permeability of cement paste using the generated 3D pore network. One of the main disadvantages 
of the use of digitalized microstructure for simulating transport properties is that the simulation results are highly dependent on the image resolution (Abyaneh et al. 2013). In general, a higher resolution can result in a more accurate simulation result. However, regarding transport properties of cement paste, it was demonstrated that the finest resolution must be optimised based on the comparison between experiment and various physical length scales of cement paste, not necessarily depend on the best resolution (Garboczi and Bentz 2001). The main purpose of this study is to obtain the 3D pore network using the MCP method for predictions of transport properties and understand the transport phenomena. It is still possible to estimate the effects of different variables on transport properties of cement paste by keeping the key parameters related to digital microstructure such as resolution and REV constant. Fig. 7 shows the simulated ionic concentration and fluid velocity distribution in cement paste under steady state, corresponding to the generated microstructure illustrated in Fig. 5(c).
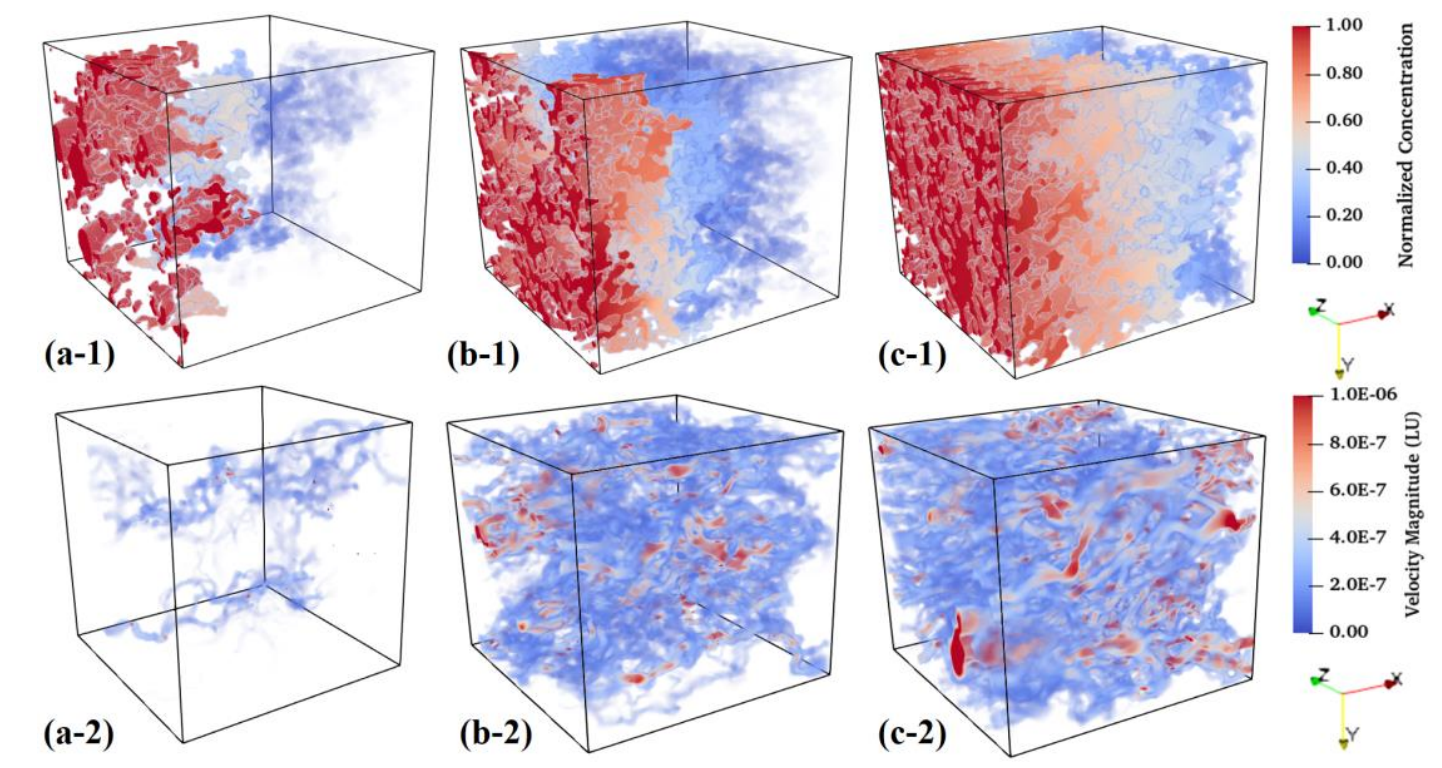

Fig. 7. Steady-state ionic concentration distribution (a-1, b-1 and c-1) and fluid velocity (a-2, b-2 and c-2) in cement paste with $w / c$ ratios of $0.5,0.6$ and 1.0 along $x$-direction.

Fig. 8(a) shows the simulated ionic diffusivity and water permeability in cement paste with $w / c$ ratios of 0.5, 0.6 and 1.0, in comparison with experimental data obtained from literature (Care 2008; Grasley et al. 2007; Hamami et al. 2012; Li et al. 2016; Li and Xu 2019; Ngala et al. 1995; Numata et al. 1990; Phung et al. 2013; Poupard et al. 2004; Rose and Grasley 2017; Tracz 2016; Zheng et al. 2018). Generally, the ionic diffusivity of cement paste is measured through chloride diffusion using the spectrophotometric technique (Ngala et al. 1995), colourimetric method (Care 2008), and electrical field acceleration method (Zheng et al. 2018; Poupard et al. 2004), while fluid permeability are referred to water permeability and gas permeability measured using dynamic pressurization 
technique (Grasley et al. 2007; Rose and Grasley 2017;), pressure-induced method (Hamami et al. 2012; Li and Xu 2019; Hamami et al. 2012), and constant water-flow method (Phung et al. 2013). As can be seen, both ionic diffusivity and fluid permeability of cement paste increase with increasing $w / c$ ratios. The increasing trend of diffusivity and permeability of cement paste displays an exponential function of $w / c$ ratio, which is consistent with the experimental and simulation results reported by Numata et al. (1990) and Zhang et al. (2014). The diffusivity and permeability of cement paste with a lower $w / c$ ratio ranging from 0.5 to 0.6 show a more dramatic increase compared to cement paste with a higher $w / c$ ratio in the range of 0.6 and 1.0. For instance, the diffusivity of cement paste with $w / c$ ratio of 0.6 is increased by two orders of magnitude from $10^{-12} \mathrm{~m}^{2} / \mathrm{s}$ to $10^{-10} \mathrm{~m}^{2} / \mathrm{s}$. As the $w / c$ ratio increases from 0.6 to 1.0 , an only 1.03 -fold increase in diffusivity from $104.97 \times 10^{-12}$ $\mathrm{m}^{2} / \mathrm{s}$ to $212.94 \times 10^{-12} \mathrm{~m}^{2} / \mathrm{s}$ can be found. Similarly, the changing trend of permeability of cement paste also indicates an exponential function with $w / c$ ratio, which agrees well with that reported in the literature (Banala and Kumar 2017). With the increase of $w / c$ ratio from 0.6 to 1.0, the effect of $w / c$ ratio on diffusivity and permeability tends to be insignificant, which can be explained by the fact that hydration products do not fill the whole capillary pore space in cement paste with a higher $w / c$ ratio. Whereas, the packing of cement particles has a significant effect, which leads to a decrease in the complexity of pore network of cement paste. For example, as seen in Fig. 5(d) and Fig. (6), the changes in porosity and pore size of cement paste with the increase of $w / c$ ratio from 0.5 to 0.6 is smaller than that with increasing $w / c$ ratio from 0.6 to 1.0 .

Besides, the simulated ionic diffusivity of cement paste shows a similar tendency to the experimental data reported in literature that there is no order of magnitude difference with the increasing $w / c$ ratio. However, the simulated water permeability exists several orders of magnitude difference compared to experimental results for cement paste with the increase of $w / c$ ratio. In general, the water permeability shows a larger scatter than those obtained from other fluids such as ethanol, gas, and so on. The literature data on water permeability is significantly lower than that obtained from ethanol with 4 69-folds (Loosveldt et al. 2002), which can be attributed to the drawback of water permeability testing. During the permeation of water, the expansion of hydration products, secondary hydration of anhydrous cement particles, and dissolution and migration of fine elements in cement paste would occur over a long period under a high-pressure gradient (Loosveldt et al. 2002). These phenomena were not considered in this study and thus the simulated water permeability tends to be higher than the experimental data obtained from literature. 
The measured gas permeability is close to the predicted result compared to water permeability, implying that gas permeability is generally higher than water permeability. During the permeation of gas, the flow velocity near the pore wall is different from that in the centre of the pores due to the interactions between gas molecules and pore walls, which was first explained first by Klinkenberg theory and defined as gas slippage (Fathi et al. 2012). In addition, the partial collision behaviour can also cause Knudsen flow, which is strongly associated with the mean free path of gas molecule and pore size (Roy et al. 2003). The collisions between gas molecules and pore walls would result in slippage and Knudsen flows, which both can increase the gas permeability. Furthermore, the difference between gas permeability and water permeability of cement paste with $w / c$ ratio of 0.5 is higher than that of cement paste with $w / c$ ratio of 0.6 , which can be explained by the fact that cement paste with a lower $w / c$ ratio has higher porosity with a small size, as shown in Fig. 6, which facilitates the collisions, leading to more slippage and Knudsen flows.

The diffusivity and permeability of cement paste are dependent on $w / c$ ratio and effective porosity (Liu et al. 2020). With the increase of effective porosity, the diffusivity and permeability are not associated with $w / c$ ratio due to the decreasing effect of cement particle packing due to cement hydration. As seen in Fig. 8(b), a more dramatic increase in both ionic diffusivity and fluid permeability can be observed in cement paste with a higher effective porosity, which can be ascribed to the more available flow paths in cement paste with a higher effective porosity. As the effective porosity increases to about 0.3 , the ionic diffusivity and fluid permeability both show a sharp increase, which can be attributed to the fact that most capillary pores are connected (Garboczi and Bentz 2001). There exists an exponential relationship between the ionic diffusivity and effective porosity of cement paste, which is consistent with the findings obtained from experiments and numerical simulations in the literature (Numata et al. 1990; Zhang et al. 2014). Regarding the change of fluid permeability with effective porosity, the fluid permeability of cement paste with a higher effective porosity (e.g. $27.56 \%$ and $36.11 \%$ ) is at least two orders of magnitude higher than that of cement paste with a lower effective porosity $(11.80 \%)$. 

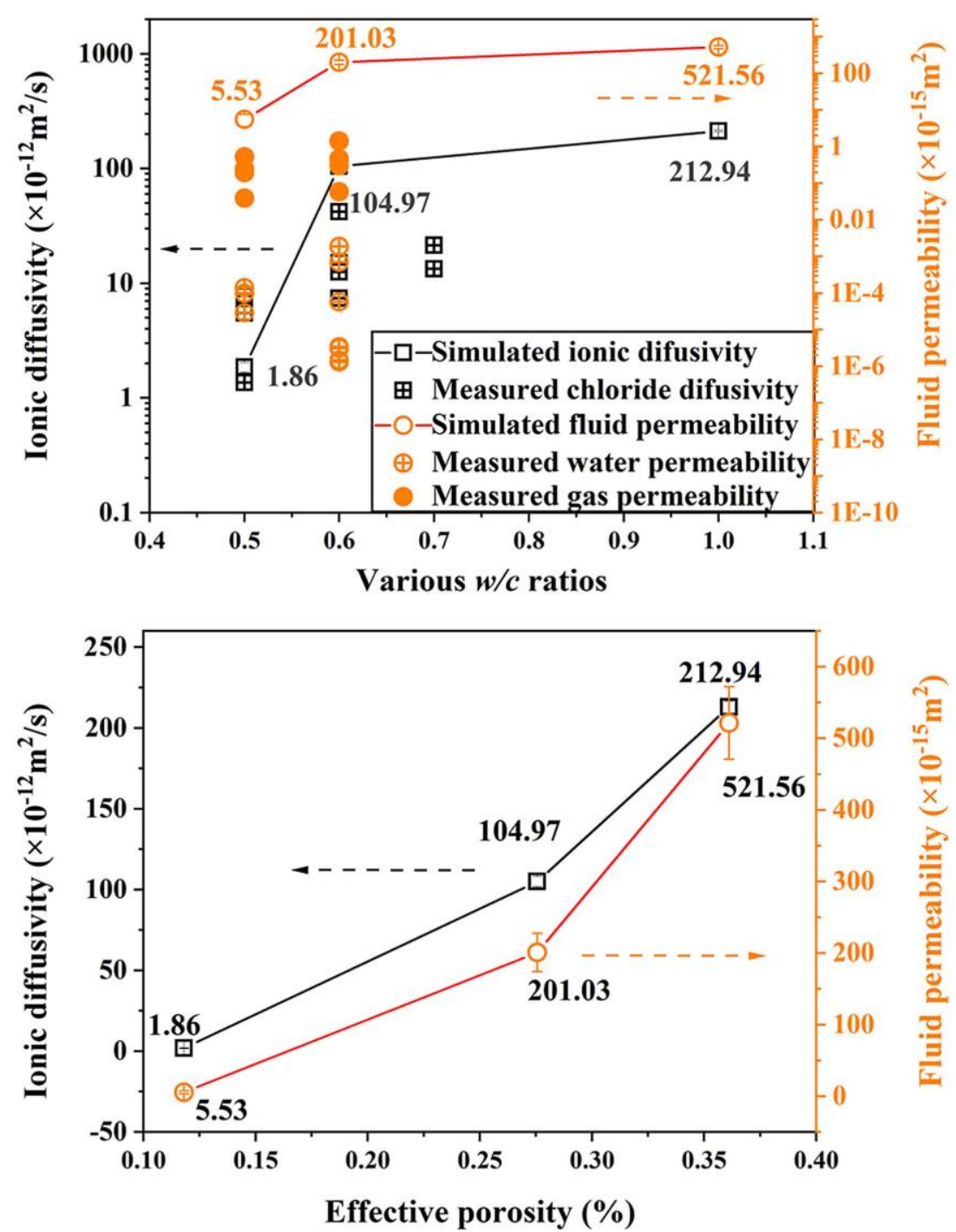

Fig. 8. Fluid permeability and ionic diffusivity in lattice unit in cement paste with (a) w/c ratios of $0.5,0.6$ and 1.0; and (b) different effective porosity along x-direction where the experimental results were collected from literature (Care 2008; Grasley et al. 2007; Hamami et al. 2012; Li et al. 2016; Li and Xu 2019; Ngala et al. 1995; Numata et al. 1990; Phung et al. 2013; Poupard et al. 2004; Rose and Grasley 2017; Tracz 2016;

Zheng et al. 2018).

\section{Conclusions}

In this study, the 3D pore network of cement paste with various $w / c$ ratios was quantitatively characterised using X-ray microtomography coupled with the metal centrifugation porosimetry (MCP) method, based on which the ionic diffusivity and fluid permeability of cement paste were simulated using lattice Boltzmann method. The simulation results of ionic diffusivity and fluid permeability of cement paste were compared with experimental data obtained from literature. The main conclusions can be drawn as follows:

1. X-ray microtomography coupled with the MCP method was successfully employed to investigate the $3 \mathrm{D}$ pore network in hardened cement paste in a quantitative manner. The contrast between pores 
and solid phases in X-ray microtomography images was effectively improved due to the heavier elements contained in the metal alloy. According to the obtained 3D pore structure, the porosity of cement paste with $\mathrm{w} / \mathrm{c}=0.5,0.6$ and 1.0 was found to be $15.21 \%, 27.81 \%$ and $36.45 \%$, respectively, while the main pore size was in the range of 0 to $20 \mu \mathrm{m}$.

2. The changes of ionic diffusivity and fluid permeability of cement paste with $w / c$ ratio exhibited a similar tendency. They both increased with the increasing $w / c$ ratio and effective porosity. The ionic diffusivity and fluid permeability of cement paste with a lower $w / c$ ratio ranging from 0.5 to 0.6 both showed a more dramatic increase.

3. With the increase of $w / c$ ratio from 0.6 to 1.0 , the effects of $w / c$ ratio on ionic diffusivity and fluid permeability were not significant, which can be attributed to the fact that the hydration products did not sufficiently fill in the whole capillary pore space for cement paste with a higher $w / c$ ratio, which decreases the complexity of pore network of cement paste.

\section{Data Availability Statement}

Some or all data, models, or code that support the findings of this study are available from the corresponding author upon reasonable request.

\section{Acknowledgements}

The financial support provided by the National Key Research and Development Program of China (NO. 2019YFC1904904) and Natural Science Foundation of China (51878153 and 51808189) is gratefully acknowledged. M. Zhang gratefully acknowledges the financial support from the Engineering and Physical Sciences Research Council (EPSRC), UK under Grant No. EP/R041504/1 and the Royal Society, UK under Award No. IEC \NSFC $\backslash 191417$ as well as the Visiting Researcher Fund Program of State Key Laboratory of Water Resources and Hydropower Engineering Science, China under Award No. 2019SGG01.

\section{Conflict of Interest}

The authors declare no conflict of interest.

\section{References}

Abyaneh, S. D., Wong, H., and Buenfeld, N. (2013). "Modelling the diffusivity of mortar and concrete using a three-dimensional mesostructure with several aggregate shapes." Computational Materials Science, 78, 63-73.

Attari, A., McNally, C., and Richardson, M. (2016). "A combined SEM-Calorimetric approach for assessing hydration and porosity development in GGBS concrete." Cement and Concrete 
Composites, 68, 46-56.

Banala, A., and Kumar, A. (2017). "Numerical simulations of permeability of plain and blended cement pastes." International Journal of Advances in Engineering Sciences and Applied Mathematics, 9(2), 67-86.

Yang, L., Liu, G., Gao, D., and Zhang, C. "Experimental study on water absorption of unsaturated concrete: w/c ratio, coarse aggregate and saturation degree". Construction and Building Materials, 2021, 272:121945

Care, S. (2008). "Effect of temperature on porosity and on chloride diffusion in cement pastes." Construction and Building Materials, 22(7), 1560-1573.

De Winter, D., Schneijdenberg, C., Lebbink, M., Lich, B., Verkleij, A., Drury, M., and Humbel, B. (2009). "Tomography of insulating biological and geological materials using focused ion beam (FIB) sectioning and low-kV BSE imaging." Journal of Microscopy, 233(3), 372-383.

Desbois, G., Hemes, S., Laurich, B., Houben, E., Klaver, J., Hoehne, N., Urai, J., Viggiani, G., and Bésuelle, P. (2016). "Investigation of microstructures in naturally and experimentally deformed reference clay rocks using innovative methods in scanning electron microscopy." $21,1-14$.

Esfandiari, J., and Loghmani, P. (2019). "Effect of perlite powder and silica fume on the compressive strength and microstructural characterization of self-compacting concrete with lime-cement binder." Measurement, 147.

Fathi, E., Tinni, A., and Akkutlu, I. (2012). "Correction to Klinkenberg slip theory for gas flow in nano-capillaries." International Journal of Coal Geology, 103, 51-59.

Garbalinska, H., Kowalski, S., and Staszak, M. (2013). "Moisture diffusivity in mortars of different water-cement ratios and in narrow ranges of air humidity changes." International Journal of Heat and Mass Transfer, 56(1-2), 212-222.

Garboczi, E.J., and Bentz, D. P. (1992). "Computer simulation of the diffusivity of cement-based materials." Journal of Materials Science, 27 (8), 2083-2092.

Garboczi, E. J., and Bentz, D. P. (2001). "The effect of statistical fluctuation, finite size error, and digital resolution on the phase percolation and transport properties of the NIST cement hydration model." Cement and Concrete Research, 31(10), 1501-1514.

Ghasemzadeh, F., and Pour-Ghaz, M. (2015). "Effect of damage on moisture transport in concrete." Journal of Materials in Civil Engineering, 27(9), 04014242. 
Grasley, Z., Scherer, G., Lange, D., and Valenza, J. (2007). "Dynamic pressurization method for measuring permeability and modulus: II. cementitious materials." Materials and Structures, 40(7), 711-721.

Hamami, A., Turcry, P., and Ait-Mokhtar, A. (2012). "Influence of mix proportions on microstructure and gas permeability of cement pastes and mortars." Cement and Concrete Research, 42(2), 490-498.

He, X., Zou, Q., Luo, L.-S., and Dembo, M. (1997). "Analytic solutions of simple flows and analysis of nonslip boundary conditions for the lattice Boltzmann BGK model." Journal of Statistical Physics, 87(1-2), 115-136.

Hellmuth, K., Siitari-Kauppi, M., Klobes, P., Meyer, K., and Goebbels, J. (1999). "Imaging and analyzing rock porosity by autoradiography and $\mathrm{Hg}$-porosimetry/X-ray computertomography-applications." Physics and Chemistry of the Earth Part a-Solid Earth and Geodesy, 24(7), 569-573.

Hou, D., Li, D., Hua, P., Jiang, J., and Zhang, G. (2019). "Statistical modelling of compressive strength controlled by porosity and pore size distribution for cementitious materials." Cement and Concrete Composites, 96, 11-20.

Jafari Azad, V., Erbektas, A. R., Qiao, C., Isgor, O. B., and Weiss, W. J. (2019). "Relating the formation factor and chloride binding parameters to the apparent chloride diffusion coefficient of concrete." Journal of Materials in Civil Engineering, 31(2), 04018392.

Jeong, N., Choi, D. H., and Lin, C.-L. (2008). "Estimation of thermal and mass diffusivity in a porous medium of complex structure using a lattice Boltzmann method." International Journal of Heat and Mass Transfer, 51(15-16), 3913-3923.

Kanit, T., Forest, S., Galliet, I., Mounoury, V., and Jeulin, D. (2003). "Determination of the size of the representative volume element for random composites: statistical and numerical approach." International Journal of Solids and Structures, 40(13-14), 3647-3679.

Kaufmann, J. (2010). "Pore space analysis of cement-based materials by combined Nitrogen sorption - Wood's metal impregnation and multi-cycle mercury intrusion." Cement and Concrete Composites, 32(7), 514-522.

Klobes, P., Riesemeier, H., Meyer, K., Goebbels, J., and Hellmuth, K. (1997). "Rock porosity determination by combination of X-ray computerized tomography with mercury porosimetry." Fresenius Journal of Analytical Chemistry, 357(5), 543-547. 
Kumar, R., and Bhattacharjee, B. (2003). "Study on some factors affecting the results in the use of MIP method in concrete research." Cement and Concrete Research, 33(3), 417-424.

Lapham, D., and Lapham, J. (2019). "BET surface area measurement of commercial magnesium stearate by krypton adsorption in preference to nitrogen adsorption." International Journal of Pharmaceutics, 568.

Li, B., Mao, J., Nawa, T., and Han, T. (2017). "Mesoscopic damage model of concrete subjected to freeze-thaw cycles using mercury intrusion porosimetry and differential scanning calorimetry (MIP-DSC)." Construction and Building Materials, 147, 79-90.

Li, K., Stroeven, M., Stroeven, P., and Sluys, L. (2016). "Investigation of liquid water and gas permeability of partially saturated cement paste by DEM approach." Cement and Concrete Research, 83, 104-113.

Li, L., Zhang, H., Guo, X., Zhou, X., Lu, L., Chen, M., and Cheng, X. (2019). "Pore structure evolution and strength development of hardened cement paste with super low water-to-cement ratios." Construction and Building Materials, 227.

Li, X., and Xu, Y. (2019). "Microstructure-Based Modeling for Water Permeability of Hydrating Cement Paste." Journal of Advanced Concrete Technology, 17(7), 405-418.

Li, Y., Guo, W., and Li, H. (2017). "Method to Calculate Cement Content in Hardened Concrete Based on Theory of Carbonization." ACI Materials Journal, 114(2), 245-251.

Liu, C., Qian, C., Qian, R., Liu, Z., Qiao, H., and Zhang, Y. (2019). "Numerical prediction of effective diffusivity in hardened cement paste between aggregates using different shapes of cement powder." Construction and Building Materials, 223, 806-816.

Liu, C., Liu, Z., and Zhang, Y. (2020). "A multi-scale framework for modelling effective gas diffusivity in dry cement paste: Combined effects of surface, Knudsen and molecular diffusion." Cement and Concrete Research, 131.

Liu, C., Wang, F., and Zhang, M. (2020). "Modelling of 3D microstructure and effective diffusivity of fly ash blended cement paste." Cement and Concrete Composites, 110.

Liu, G., Zhang, Y., Ni, Z., and Huang, R. (2016). "Corrosion behavior of steel submitted to chloride and sulphate ions in simulated concrete pore solution." Construction and Building Materials, $115,1-5$.

Loosveldt, H., Lafhaj, Z., and Skoczylas, F. (2002). "Experimental study of gas and liquid permeability of a mortar." Cement and Concrete Research, 32(9), 1357-1363. 
Moro, F., and Böhni, H. (2002). "Ink-bottle effect in mercury intrusion porosimetry of cement-based materials." Journal of Colloid and Interface Science, 246(1), 135-149.

Ngala, V., Page, C., Parrott, L., and Yu, S. (1995). " Diffusion in cementitious materials: II, further investigations of chloride and oxygen diffusion in well-cured OPC and OPC/30\% PFA pastes." Cement and Concrete Research, 25(4), 819-826.

Numata, S., Amano, H., and Minami, K. (1990). " Diffusion of tritiated water in cement materials." Journal of Nuclear Materials, 171(2-3), 373-380.

Oslakovic, I.S., Bjegovic, D., and Mikulic, D. (2010). "Evaluation of service life design models on concrete structures exposed to marine environment." Materials and Structures, 43(10), 13971412.

Pan, C., Luo, L.-S., and Miller, C. T. (2006). "An evaluation of lattice Boltzmann schemes for porous medium flow simulation." Computers \& fluids, 35(8-9), 898-909.

Patel, R.A., Phung, Q.T., Seetharam, S.C., Perko, J., Jacques, D., Maes, N., De Schutter, G., Ye, G., and Van Breugel, K. (2016). "Diffusivity of saturated ordinary Portland cement-based materials: A critical review of experimental and analytical modelling approaches." Cement and Concrete Research, 90, 52-72.

Phung, Q., Maes, N., De Schutter, G., Jacques, D., and Ye, G. (2013). "Determination of water permeability of cementitious materials using a controlled constant flow method." Construction and Building Materials, 47, 1488-1496.

Pichler, B., Hellmich, C., Eberhardsteiner, J., Wasserbauer, J., Termkhajornkit, P., Barbarulo, R., and Chanvillard, G. (2013). "Effect of gel-space ratio and microstructure on strength of hydrating cementitious materials: An engineering micromechanics approach." Cement and Concrete Research, 45, 55-68.

Poupard, O., Ait-Mokhtar, A., and Dumargue, P. (2004). "Corrosion by chlorides in reinforced concrete: Determination of chloride concentration threshold by impedance spectroscopy." Cement and Concrete Research, 34(6), 991-1000.

Qian, R., Zhang, Y., Liu, C., Yang, L., Liu, G., and She, W. (2018). "Quantitative characterization of three-dimensional pore structure in hardened cement paste using X-ray microtomography combined with centrifuge driven metal alloy intrusion." Materials Characterization, 145, 277-283.

Rose, J., and Grasley, Z. (2017). "Comparison of Permeability of Cementitious Materials Obtained 
via Poromechanical and Conventional Experiments." Journal of Materials in Civil Engineering, 29(9).

Roy, S., Raju, R., Chuang, H., Cruden, B., and Meyyappan, M. (2003). "Modeling gas flow through microchannels and nanopores." Journal of Applied Physics, 93(8), 4870-4879.

Rozenbaum, O., and du Roscoat, S. (2014). "Representative elementary volume assessment of threedimensional x-ray microtomography images of heterogeneous materials: Application to limestones." Physical Review E, 89(5).

She, W., Du, Y., Zhao, G., Feng, P., Zhang, Y., and Cao, X. (2018). "Influence of coarse fly ash on the performance of foam concrete and its application in high-speed railway roadbeds." Construction and Building Materials, 170, 153-166.

Song, Y., Dai, G., Zhao, L., Bian, Z., Li, P., and Song, L. (2020). "Permeability prediction of hydrated cement paste based on its 3D image analysis." Construction and Building Materials, 247.

Song, Y., Davy, C., Troadec, D., and Bourbon, X. (2019). "Pore network of cement hydrates in a High Performance Concrete by 3D FIB/SEM - Implications for macroscopic fluid transport." Cement and Concrete Research, 115, 308-326.

Qian, R., Liu, G., Liu, Z., She, W., Qiao, H., and Zhang, Y. (2021) "Investigations on threedimensional pore-structure in cementitious materials using metal centrifugation porosimetry and simulation". Materials Letters, 282, 128684

Tang, L., and Sorensen, H. (2001). "Precision of the Nordic test methods for measuring the chloride diffusion/migration coefficients of concrete." Materials and Structures, 34(242), 479-485.

Tracz, T. (2016). "Open porosity of cement pastes and their gas permeability." Bulletin of the Polish Academy of Sciences-Technical Sciences, 64(4), 775-783.

Ukrainczyk, N., and Koenders, E. (2014). "Representative elementary volumes for 3D modeling of mass transport in cementitious materials." Modelling and Simulation in Materials Science and Engineering, 22(3), 035001.

Wang, M., Wang, J., Pan, N., and Chen, S. (2007). "Mesoscopic predictions of the effective thermal conductivity for microscale random porous media." Physical Review E, 75(3), 036702.

Willis, K., Abell, A., and Lange, D. (1998). "Image-based characterization of cement pore structure using wood's metal intrusion." Cement and Concrete Research, 28(12), 1695-1705.

Wong, H., Head, M., and Buenfeld, N. (2006). "Pore segmentation of cement-based materials from backscattered electron images." Cement and Concrete Research, 36(6), 1083-1090. 
Yio, M., Mac, M., Wong, H., and Buenfeld, N. (2015). "3D imaging of cement-based materials at submicron resolution by combining laser scanning confocal microscopy with serial sectioning." Journal of Microscopy, 258(2), 151-169.

Yio, M., Wong, H., and Buenfeld, N. (2017). "Representative elementary volume (REV) of cementitious materials from three-dimensional pore structure analysis." Cement and Concrete Research, 102, 187-202.

Yu, P., Duan, Y., Chen, E., Tang, S., and Wang, X. (2018). "Microstructure-based fractal models for heat and mass transport properties of cement paste." International Journal of Heat and Mass Transfer, 126, 432-447.

Zeng, Q., Wang, X., Yang, P., Wang, J., and Zhou, C. (2019). "Tracing mercury entrapment in porous cement paste after mercury intrusion test by X-ray computed tomography and implications for pore structure characterization." Materials Characterization, 151, 203-215.

Zhang, M., Ye, G., and van Breugel, K. (2012). "Modeling of ionic diffusivity in non-saturated cement-based materials using lattice Boltzmann method." Cement and Concrete Research, 42(11), 1524-1533.

Zhang, M., Ye, G., and van Breugel, K. (2013). "Microstructure-based modeling of permeability of cementitious materials using multiple-relaxation-time lattice Boltzmann method." Computational Materials Science, 68, 142-151.

Zhang, M., Ye, G., and van Breugel, K. (2014). "Multiscale lattice Boltzmann-finite element modelling of chloride diffusivity in cementitious materials. Part II: Simulation results and validation." Mechanics Research Communications, 58, 64-72.

Zheng, J., Zhang, J., Zhou, X., Wu, Y., Ye, Y., and Wang, Y. (2018). "A three-step analytical scheme for estimating the steady-state chloride diffusion coefficient of mature cement paste." Construction and Building Materials, 191, 1004-1010. 